\title{
A Case of Solitary Hepatic Cyst with Accessory Sulci of Liver
}

\author{
APARNA MURALEEDHARAN', YOGESH ASHOK SONTAKKE², PARKASH CHAND ${ }^{\circ}$
}

\section{ABSTRACT}

Hepatic cystic lesions are rare accidental findings on imaging techniques and present a diagnostic challenge to the treating physician. They may range from a simple serous cyst to a complex heterogenous malignant cyst. However, the incidence of hepatic cysts is very less and hence is usually overlooked in radiological studies. Accessory grooves or fissures are frequently found on the hepatic surface and can cause diagnostic dilemma among radiologists and surgeons. Some accessory fissures might serve as guides during hepatectomy as they may separate hepatic segments. During routine dissection, we found a peculiar liver with a single cystic lesion filled with a white cheesy thick viscous material, several accessory fissures and an abnormal tissue band bridging the fissure for ligamentum teres hepatis. Histological study of the cyst wall and the abnormal band was also performed using routine and special staining techniques. The current report could alert radiologists and surgeons about various causes, radiological findings and surgical appearance of some asymptomatic liver cysts. Common variations in the external appearance and anomalies of the lobes and fissures of the liver are also reviewed here; the knowledge of which may minimize misdiagnosis or over diagnosis of liver diseases.

\section{CASE REPORT}

The current case report is about a peculiar liver obtained from an adult male cadaver during routine dissection for medical undergraduates.

The liver was firm to hard in consistency. Some areas of the liver appeared discoloured. Histologically, liver tissue showed fatty degeneration. A few cells resembled adipocytes and had empty cytoplasm and peripherally pushed flat nucleus. The discoloured area showed congestion, i.e., presence of numerous RBCs in the liver sinusoids as compared to other areas. This area also showed vacuolated cells.

A Solitary cyst was present on superior surface at its junction with anterior and right surface and was white in colour, $2.3 \times 2.2$ $\mathrm{cm}$ size and circular [Table/Fig-1a]. On section, it was unilocular and was filled with a thick white cheesy material [Table/Fig-1b,c]. Depth of the cyst was $2.2 \mathrm{~cm}$ [Table/Fig-1b]. On histological examination, the cyst wall was thick and well-circumscribed well separated from adjoining liver tissue by connective tissue. Cyst wall was lined by simple squamous/cuboidal epithelium (that separated off) with sub-epithelial eosinophilic homogenous material and numerous blood vessels [Table/Fig-2a,b]. Adjoining liver parenchyma showed presence of vacuolated cells and was congested. Masson's trichrome staining was done for the cyst wall which showed two distinct layers-inner or fibrous layer with thickness of $92.98 \mu \mathrm{m}$, outer or cellular layer with thickness of $88.69 \mu \mathrm{m}$ [Table/Fig-2c,d].

Fissure for ligamentum teres made a $2.5 \mathrm{~cm}$ deep notch on the inferior border of liver with a band of liver parenchyma bridging the fissure for ligamentum teres [Table/Fig-1d]. Fissure for ligamentum teres was found to be deep [Table/ Fig-1e]. The band was $2 \mathrm{~cm}$ wide, between quadrate lobe and left lobe, $4 \mathrm{~cm}$ posterior to inferior border, $2 \mathrm{~cm}$ anterior to porta hepatis. On microscopy, the band showed presence of liver tissue and some peritoneal extension [Table/Fig-2e]. Ligamentum teres was separated from the liver tissue by loose areolar tissue and covered by peritoneum which also contained the paraumbilical vein.

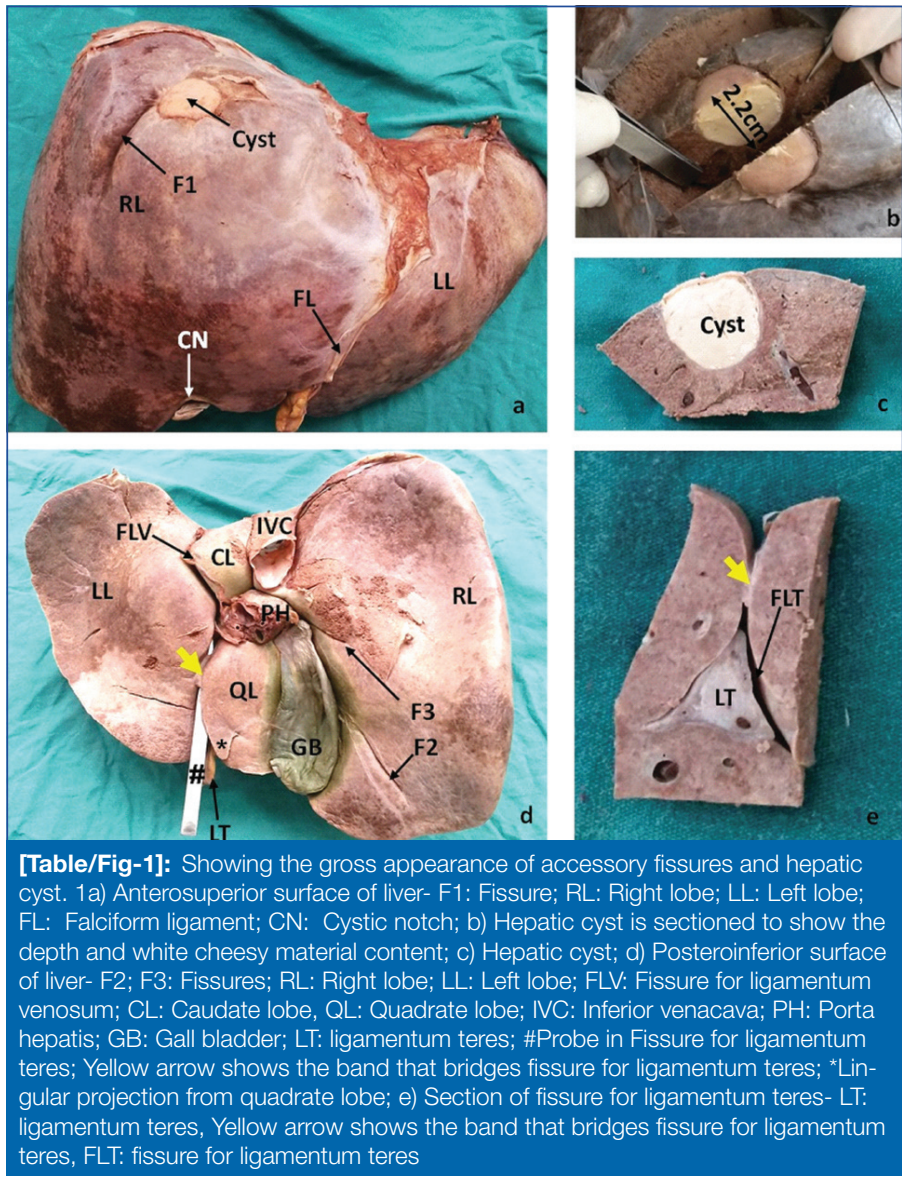

There was a lingular projection from the quadrate lobe on anterior and left end of quadrate lobe adjacent to fissure for ligamentum teres separated from right part of quadrate lobe by $1.5 \mathrm{~cm}$ long fissure. It was triangular with $1.2 \mathrm{~cm}$ base and was $1.5 \mathrm{~cm}$ long [Table/Fig-1d].

Three accessory fissures were noted on the surface of liver. A vertical fissure F1 $6.5 \mathrm{~cm}$ long [Table/Fig-1a] extending from superior to anterior surface separating anterior surface from right surface, an 
oblique fissure F2 on inferior surface of right lobe [Table/Fig-1d] on pyloric area $4 \mathrm{~cm}$ long, $4 \mathrm{~mm}$ deep and $1.3 \mathrm{~cm}$ to the right of gall bladder fossa and an oblique fissure F3 on inferior surface of right lobe extending to right from right end of porta hepatis $3 \mathrm{~cm}$ long 1 cm deep [Table/Fig-1d].

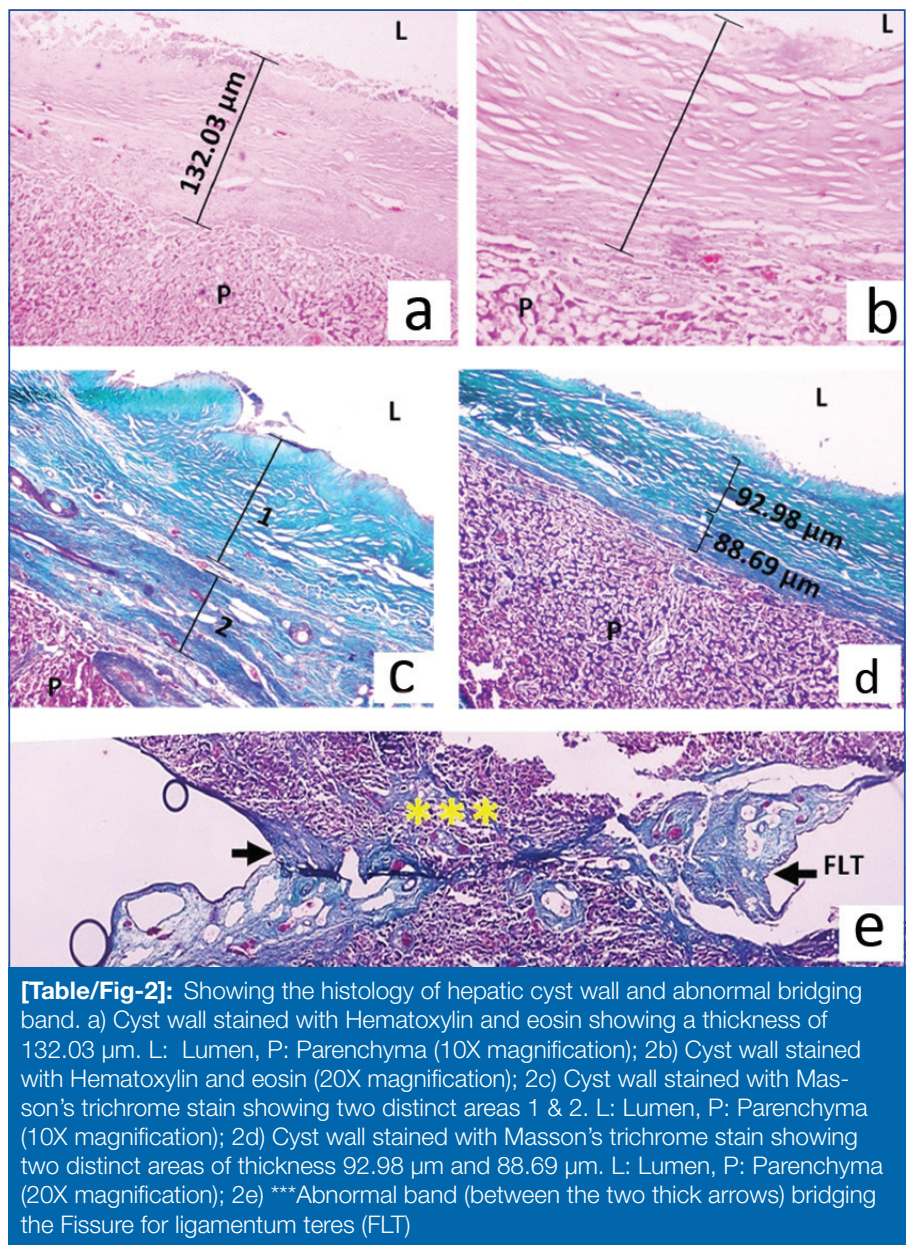

\section{DISCUSSION}

The current case describes a solitary cyst associated with accessory fissures on the surface of a liver which occurring together is rare and has not been reported in the past. The liver is divided into anatomical right and left lobes by the attachments of the falciform ligament, fissure for ligamentum teres and ligamentum venosum [1]. Major fissures on the liver surface divide the liver into the named anatomical lobes. Minor or accessory grooves are frequently found on the hepatic surface [2]. These could be congenital, developmental or acquired due to diaphragmatic or costal pressure. The absence of normal fissures or the presence of additional lobes and fissures might lead to confusion in diagnosis [3].

The liver is known to show lobe and fissure anomalies. The fissures have various lengths and shapes and could be single or multiple. The existence of additional lobes, fissures or bands and the absence or underdevelopment of normal lobes and fissures can lead to difficulties during surgery, autopsy or clinical misdiagnosis. Several lobe and fissure anomalies of the liver have been noted and reported in the past. Joshi SD et al., reported presence of abnormal notching in the lower border of the caudate lobe in $18 \%$ of livers, presence of vertical fissures in 30\%, and abnormally well-developed papillary process in $32 \%$ of livers in their study on variations of the liver [4]. Preoperative imaging and detection of abnormal lobes has even led to misdiagnosis of lesser omental lymphadenopathy which would alter the management as reported by Fitzgerald $\mathrm{R}$ et al., [5]. The gall bladder, cystic notch and the hepatobiliary tree can also show variations [6]. Literature revealed that majority of the accessory fissures was located on the diaphragmatic surface of liver and most of these had been detected during radiological investigations for other purposes. A higher frequency of the diaphragmatic sulci is attributed to the costal pressure exerted by the ribs and the diaphragm. The embryological basis of the morphological anomalies of liver appearing during the process of organogenesis is still unclear and needs to be studied more. Other congenital malformations of the liver include agenesis or absence of lobes or segments, deformed lobes, atrophic or hypoplastic lobes [7-9].

Major or minor grooves of the liver as seen in the present case may play a role in hepatectomy [3]. For instance, accessory fissures may act as guidance during segmental hepatectomy by coinciding with a branch of the portal vein [10]. These grooves can be used not only as a clue for localization of a structure but also for making a decision regarding the resection margin in hepatectomy. Therefore, detailed investigation of hepatic fissures and other structural anomalies is significant and helpful in the anatomic evaluation and surgical understanding of the liver.

Hepatic cystic lesions represent a heterogeneous group of conditions with different etiology, symptoms, diagnostic findings and treatment, the commonest being simple cysts with an incidence of 2.5-18\% [11]. They are predominantly asymptomatic and are found as a mere coincidence on radiological investigations for vague or unrelated symptoms [11]. Most of the lesions have no clinical significance [12]. Symptomatic hepatic cysts usually present with a palpable abdominal mass or hepatomegaly. A small proportion of patients present with abdominal pain, early satiety, nausea and vomiting as a result of a mass effect. Complications such as haemorrhage and obstructive jaundice are possible in larger cysts. The most important factors in the development of complications are the number, size and location of cysts, and their relationship with adjacent organs and blood vessels. Differential diagnosis of cystic lesions of liver is Monocytic disease (which includes simple cyst, cystic echinococcosis, alveolar echinococcosis, cystadenoma and cystadenocarcinoma) and Polycystic disease (which includes autosomal dominant polycystic kidney disease and autosomal dominant polycystic liver disease). Simple cysts, the most common cystic liver disease arise from aberrant bile ducts that dilate as a result of obstruction and stasis and are filled with a yellowish odourless fluid. Other cystic conditions include abscesses, bile duct hamartoma or von Meyenburg complexes, Caroli disease [5] and metastatic cysts [13]. These cysts often present a diagnostic challenge and it is important to distinguish a benign lesion from a malignant one to facilitate treatment.

Lantinga MA et al., and Borhani AA et al., have published reviews on evaluation and differential diagnosis of cystic hepatic lesions $[11,14]$. Borhani AA et al., has provided an algorithm that is useful for radiologists, physicians and surgeons for diagnosis and management of hepatic cysts. Radiological findings like simple morphology, complex morphology, enhancing central dot sign and no enhancement on administration of IV contrast combined with clinical history of the patient like history of trauma or surgery, history of pancreatitis, subcapsular location of cysts, extrahepatic malignancy, cirrhosis, endemicity, infection, immunosuppression, irregularity and periportal location can help in accurate diagnosis of the condition [14]. Mavilia MG et al., found that cyst features in non-invasive investigations like ultrasound, contrast enhanced ultrasound, CT scan, contrast enhanced CT scan and MRI coupled with clinical features and enzyme levels of SGPT, SGOT, GGT and CA 19-9 will help to reach at an accurate diagnosis so as to plan treatment [15]. In the present case, the cyst was a single thickwalled cyst filled with a cheesy white material which is against the appearance of a simple cyst. Because of the small size and superficial location, it would have remained asymptomatic and undiagnosed. In such a case, histological examination would throw some light on to the diagnosis and hence was done by staining with routine Haematoxylin and eosin stain and by using Masson's trichrome special stain. The cheesy material and histological examination of 
the cyst shows that the most likely diagnosis could be that of an epidermoid cyst which is very rare.

\section{CONCLUSION}

During routine dissection, an anatomically variant liver was found with a rare type of solitary cyst filled with a cheesy material and few accessory fissures and abnormal lobes. Hepatic cysts have varied pathology and clinical implications. Radiologists and surgeons should be aware of the possibility of accessory fissures and asymptomatic hepatic cysts when approaching a patient with liver pathology so as to reduce chances of misdiagnosis and to assist in planning appropriate surgical approaches.

\section{REFERENCES}

[1] Standring S. Gray's Anatomy: The Anatomical Basis of Clinical Practice.40 $0^{\text {th }}$ ed. Edinburgh: Churchill Livingstone; 2008.

[2] Carrim ZI, Murchison JT. The prevalence of simple renal and hepatic cysts detected by spiral computed tomography. Clin Radiol. 2003;58:626-29.

[3] Lee JH, Lee SY, Hwang DW, Park KM, Lee YJ. Practical usefulness of minor grooves of the hepatic surface for liver resection. Hepato-Gastroenterology. 2012;59:458-60.

[4] Joshi SD, Joshi SS, Athavale SA. Some interesting observations on the surface features of the liver and their clinical implications. Singapore Med J. 2009;50:715-19.
[5] Fitzgerald R, Hale M, Williams CR. Case report: Accessory lobe of the liver mimicking lesser omental lymphadenopathy. Br J Radiol. 1993;66:839-41.

[6] Ogawa T, Ohwada S, Ikeya T, Shiozaki H, Aiba S, Morishita Y. Left -sided gallbladder with anomalies of the intrahepatic portal vein and anomalous junction of the pancreaticobiliary ductal system: A case report. Hepatogastroenterology. 1995:42:645-49.

[7] Llorente J, Dardik H. Symptomatic accessory lobe of the liver associated with absence of the left lobe. Arch Surg. 1971:102:221-23.

[8] Othman FB, Latiff AA, Suhaimi FH, Das S. Accessory sulci of the liver: An anatomical study with clinical implications. Saudi Med J. 2008;29:1247-49.

[9] Rao PPJ, Murlimanju BV, Lobo SW, Menezes RG. An unusual morphology of the human liver: A case report with emphasis on its clinical implications. J Morphol Sci. 2011;28:303-04.

[10] Sreekanth T. Diaphragmatic fissures and accessory sulci of liver in the population of Telangana. Int J Anat Res. 2016;4:3273-78.

[11] Lantinga MA, Gevers TJG, Drenth JPH. Evaluation of hepatic cystic lesions. World J Gastroenterol. 2013;19:3543-54.

[12] Muktyaz H, Nema U, Suniti MR, Mahboobul H. Anatomical study of accessory sulci of liver and its clinical significance in North Indian Population. Int J Med Health Sci. 2013;2:224-29.

[13] Fiori S, Gobbo1 AD, Gaudioso G, Caccamo L, Massironi S, Cavalcoli F, et al. Hepatic pseudocystic metastasis of well-differentiated ileal neuroendocrine tumor: A case report with review of the literature. Diagnostic Pathology. 2013;8:148-53.

[14] Borhani AA, Wiant A, Heller MT. Cystic hepatic lesions: A Review and an algorithmic approach. AJR. 2014;203:1192-204.

[15] Mavilia MG, Pakala T, Molina M, Wu GY. Differentiationg cystic liver lesions: A review of imaging modalities, diagnosis and management. J Clin Transpl Hepatol. 2018;6:208-16.

PARTICULARS OF CONTRIBUTORS:

1. Assistant Professor, Department of Anatomy, Pondicherry Institute of Medical Sciences, Puducherry, India.

2. Associate Professor, Department of Anatomy, Jawaharlal Institute of Postgraduate Medical Education and Research, Puducherry, India

3. Professor (Retired), Department of Anatomy, Jawaharlal Institute of Postgraduate Medical Education and Research, Puducherry, India.

NAME, ADDRESS, E-MAIL ID OF THE CORRESPONDING AUTHOR:

Dr. Yogesh Ashok Sontakke,

Associate Professor, Department of Anatomy, Jawaharlal Institute of Postgraduate Medical Education and Research (JIPMER),

Dhanvantari Nagar, Puducherry-605006, India.

E-mail: dryogeshas@rediffmail.com

Date of Submission: Jun 15, 2019 Date of Peer Review: Jul 03, 2019

Date of Acceptance: Jul 11, 2019

FINANCIAL OR OTHER COMPETING INTERESTS: None. 